American Journal of Infectious Diseases 5 (1): 11-16, 2009

ISSN 1553-6203

(C) 2009 Science Publications

\title{
Serological Evaluation of Brucella abortus S99 Lipopolysaccharide Extracted by an Optimized Method
}

\author{
${ }^{1,2}$ Ali Sharifat Salmani, ${ }^{1}$ Seyed Davar Siadat, ${ }^{2}$ Mohammad Reza Fallahian, ${ }^{1}$ Hojat Ahmadi, \\ ${ }^{1}$ Dariush Norouzian, ${ }^{2}$ Parichehr Yaghmai, ${ }^{1}$ Mohammad Reza Aghasadeghi, \\ ${ }^{1}$ Jalal Izadi Mobarakeh, ${ }^{1}$ Seyed Mehdi Sadat, ${ }^{3}$ Mehrangize Zangeneh and ${ }^{4}$ Maryam Kheirandish \\ ${ }^{1}$ Pasteur Institute of Iran, Tehran 13164, Iran \\ ${ }^{2}$ Islamic Azad University, Science and Research Branch, Tehran, Iran \\ ${ }^{3}$ Faculty of Medicine, Islamic Azad University, Tehran, Iran \\ ${ }^{4}$ Research Center of Iranian Blood Transfusion Organization, Tehran, Iran
}

\begin{abstract}
Problem statement: Brucellosis is a globally found infectious disease and there is no licensed vaccine against human brucellosis. The present study carried-out to evaluate the potency of our modified extracted lipopolysaccharide (LPS) of B. abortus to elicit specific anti-Brucella antibodies in animal model (Rabbit) as a part of a candidate vaccine for brucellosis. Lipopolysaccharide is one of the main virulence factors and the most immunogenic structure of smooth strains of Brucella. Approach: Lipopolysaccharide of B. abortus S99 (S-LPS) initially extracted through an optimized method as described previously. After biochemical and pyrogenicity evaluations of the extracted S-LPS humoral immune response against the extracted LPS analyzed in animal model through serological assays such as Rose Bengal assay, Rapid agglutination (Rapid Wright) test and Standard agglutination test (SAT or Wright test) to demonstrate the specific elicited antibodies against the injected LPS. In addition, the interaction of LPS and anti-LPS antibodies was demonstrated by Agarose Gel Immunodiffusion (AGID) assay. Results: Higher doses of B. abortus S99 LPS caused less or equal body temperature increase in comparison to E. coli LPS doses. Sera of immunized animals had been reported positive by RBT because of $B$. abortus LPS immunogenicity which we extracted through our optimized method. The highest titer of anti-Brucella antibodies detected two weeks after the third immunization (assayed by rapid slide agglutination and standard agglutination tests). Anti-Brucella antibodies of immunized animals reacted more specifically with the LPS of B. abortus in comparison with E. coli LPS and precipitation lines between B. abortus LPS and immune sera appeared after $30 \mathrm{~min}$ while detected after three hours for $E$. coli LPS. Conclusions/Recommendations: The properties of B. abortus S99 LPS concluded from the present study results, suggest the possible use of this component as a carrier or a part of a sub-unit or conjugated vaccine for human brucellosis.
\end{abstract}

Key words: Brucella abortus, Lipopolysaccharide, Agarose gel-immunodiffusion (AGID), Rose Bengal Test (RBT), Rapid agglutination test, Standard Agglutination Test (SAT)

\section{INTRODUCTION}

Brucella abortus is a bacterium that can cause abortions in cattle and a debilitating fever (undulant fever) that may persist intermittently for years in humans. This disease is worldwide, with areas of high endemicity such as the Mediterranean, Middle East, Latin America and Asia ${ }^{[1,2]}$. The incidence in humans ranges widely between different regions, with values of up to 200 cases per 100,000 populations.

Human brucellosis has a great variety of clinical manifestations, making it difficult to diagnose clinically. Therefore, the diagnosis must be confirmed by isolation of Brucella, mostly from blood culture or by the detection of an immune response to its antigens such as lipopolysaccharide (LPS). The diagnosis of brucellosis based exclusively on Brucella isolation presents several drawbacks. The slow growth of Brucella in primocultures may delay diagnosis for more than 7 days $^{[3,4]}$. Also, blood culture sensitivity is often low, ranging from (50-90\%) depending on disease stage, Brucella species, culture medium, quantity of circulating bacteria and the blood culture technique employed $^{[5,6]}$. Hence, serological tests play a major role Corresponding Author: Seyed Davar Siadat, Pasteur Institute of Iran, Tehran 13164, Iran 
in cases when the disease cannot be detected by blood culture. However, the interpretation of these tests is often difficult, particularly in patients with chronic brucellosis, in reinfections and relapses and in areas of endemicity, where a high portion of the population have antibodies against brucellosis. Many serological tests have been used for the diagnosis of human brucellosis. The most commonly used tests are the Serum Agglutination Test (SAT), the Coombs anti-Brucella test, the Rose Bengal Test (RBT) and complement fixation. During the last decade, radioimmunoassay ${ }^{[7,8]}$ and enzyme immunoassay ${ }^{[9-10]}$ tests have also been used. These present technical difficulties since they require skilled personnel and high-cost material. Also, interpretation of enzyme immunoassay results is difficult due to the variability of antigens and technical procedures employed. Among the techniques used for the diagnosis of human brucellosis, SAT and the Coombs test are most often used and their performance in disease diagnosis and during disease evolution has been studied thoroughly ${ }^{[11]}$. LPS is known as the main virulence factor of Brucella and LPS deficient strains have less virulence and intra-cellular survival potency. Agarose Gel-Immuno Diffusion (AGID) test also has been reported to be a simple and rapid assay to demonstrate antibodies elicited against Brucella antigens (especially LPS) ${ }^{[12]}$.

The LPS of $B$. abortus has been extensively evaluated and studied. Different strains of $B$. abortus are classified to rough and smooth based on the LPS they express (rough LPS and smooth LPS, respectively).Wild type Brucellae mainly express smooth LPS (S-LPS) and it is the main antigenic and immunogenic structure on the surface of smooth strains of Brucella. B. abortus S-LPS (as same as the most gram negative bacteria) consists of three domains: Lipid A, Core oligosaccharide and $\mathrm{O}$ specific polysaccharide (O-antigen) but possesses a peculiar non-classical LPS as compared with LPS of Enterobacteria such as E. coli. The lipid A of B. abortus LPS consists of special fatty acids (different from the other gram negative bacteria) and a unique structure. This moiety of $B$. abortus LPS possesses a diamino glucose backbone and the acyl groups are longer (C1819, C28) and are only linked to the core by amide bounds. Further the O-antigen of Brucella LPS is a homopolymer of perosamine (4, 6-dideoxy-4formamido-d-mannopyranosyl). Since LPS is the main expressed antigen on the surface of Brucella the serologic responses following Brucella infection are directed against LPS. Thus in human and animal, the serological diagnosis of brucellosis is mainly based on the detection of specific anti-LPS antibodies ${ }^{[13]}$.
In the present study we have initially extracted the LPS of B. abortus S99 (S-LPS) through an optimized method as described previously. After biochemical evaluations of the extracted S-LPS we analyzed the humoral immune response against the extracted LPS in animal model via serological assays such as the rose bengal assay, rapid agglutination (rapid wright) test, Serum Agglutination Test (SAT or wright test) and 2ME Wright test to demonstrate specific IgM and IgG antibodies elicited against the injected LPS. In addition, the interaction of LPS and anti-LPS antibodies demonstrated by Agarose Gel Immunodiffusion (AGID) assay.

\section{MATERIALS AND METHODS}

Bacteria strain and cellular biomass production: B. abortus S99 obtained from the collection of standard bacteria, Pasteur institute of Iran (Tehran). To achieve the cellular biomass of B. abortus S99 fermentation process carried out as previously described ${ }^{[14]}$. Briefly, Microorganism was cultured in slant Brucella agar medium (Merck) at $37 \pm 1^{\circ} \mathrm{C}$ for $72 \mathrm{~h}$ and then cultured in a $5 \mathrm{~L}$ flask containing $2 \mathrm{~L}$ Brucella broth (Merck) for $72 \mathrm{~h}$ to achieve the seed culture. Seed culture inoculated to the 60 liter industrial fermentor (Novapaljas, contactflow B.V, Netherlands) with $40 \mathrm{~L}$ working volume. Finally biomass of B. abortus S99 deactivated by (10\%) phenol $(\mathrm{w} / \mathrm{w})$ and harvested through centrifugation at $3800 \mathrm{rpm}$.

LPS Extraction procedure and chemical analysis of extracted sample: As it is previously described LPS of B. abortus $\mathrm{S} 99$ extracted by an optimized method based on hot phenol-water extraction and the extracted sample was chemically analyzed to define the content of LPS, Keto Deoxy Octanate (KDO), protein and nucleic $\operatorname{acids}^{[13]}$.

Rabbit pyrogen test: Five groups of rabbits weighing $2.5-3 \mathrm{~kg}$ were injected with $B$. abortus S99 LPS at a concentration of $100,10,1,0.1$, or $0.01 \mu \mathrm{g} \mathrm{mL}$ in $3 \mathrm{~mL} \mathrm{~kg}$ of body weight. Each group consisted of three rabbits. The baseline rectal temperature was recorded before injections and after $30 \mathrm{~min}$ the rabbits were inoculated in the ear vein. Temperatures were recorded every $\mathrm{h}$ for $3 \mathrm{~h}$. As a comparative study, pyrogenicity of $E$. coli LPS assayed by another rabbit pyrogen test as same as B. abortus S99 LPS. The test indicates the presence of a pyrogen when one of the three rabbits has an increase in body temperature higher than $0.6^{\circ} \mathrm{C}$ or when the sum of the maximum temperature rises in the three individual rabbits exceeds $1.4^{\circ} \mathrm{C}^{[15]}$. 
Immunization schedule: $10 \mu \mathrm{g} \mathrm{mL}^{-1}$ of $B$. abortus $\mathrm{S} 99$ LPS injected (intramuscular) to a group of 3 rabbits. Then animal models inoculated with booster injections in 14 and 28 days after the first injection. The animals were bled on the days 0 (before any immunization and as the negative control), 14 (before the first booster injection), 28 (before the second booster injection) and 42 (two weeks after the second injection) and the immune sera were separated, pooled and kept in $-20^{\circ} \mathrm{C}$.

Detection of synthesized antibodies against $B$. abortus S99 LPS: Presence of Anti-Brucella abortus S99 LPS in the sera of immunized animals demonstrated by Rose Bengal test (RBT), SAT (tube agglutination and rapid slide agglutination) and Agarose Gel-Immuno Diffusion (AGID) ${ }^{[16]}$.

Rose bengal test (RBT): $30 \mu \mathrm{L}$ of animal sera and $30 \mu \mathrm{L}$ of rose bengal antigen (obtained from bacterial vaccines and antigens production department of Pasteur Institute of Iran, Tehran) mixed together on a slide and gently shook for four min at the room temperature. Simultaneously a similar process done for standard antiserum (Pasteur Institute of Iran, Tehran) and finally positive serum samples (containing anti-Brucella antibodies) detected through observation of agglutination.

Rapid slide agglutination test: Serial dilution of animal serum samples made from $1 / 20-1 / 640$ on a scaled (cellular) flat slide. Each of the diluted samples mixed to a drop of standard antigen (Pasteur Institute of Iran, Tehran) and gently shook for a min. Also a drop of standard antigen was added to one of the squares of scaled slide as the negative control. The highest serum dilution showing (50\%) agglutination was considered as the titer of serum.

Standard agglutination test: Serial dilution of animal serum samples $(0.5 \mathrm{~mL})$ made from $1 / 20-1 / 5120$ in 9 different tubes. Then $0.5 \mathrm{~mL}$ of standard antigen (Pasteur Institute of Iran, Tehran) added to each sample. Also $0.5 \mathrm{~mL}$ of the standard antigen added to $0.5 \mathrm{~mL}$ of normal saline as the negative control. Samples kept at $37^{\circ} \mathrm{C}$ for $24 \mathrm{~h}$ and finally analyzed to detect the positive samples and the titer of sera in comparison to the negative control. The highest serum dilution showing $(50 \%)$ agglutination was considered as the titer of serum.

Agarose gel-immunodiffusion (AGID) test: The gel was prepared by dissolving (1.8\%) agarose in $0.1 \mathrm{M}$ Barbital buffer ( $\mathrm{pH}$ 8.6). $\mathrm{NaCl}$ had been added to a concentration of $(20 \%)$ to the barbital buffer. Prepared gel dispensed on plates at $65^{\circ} \mathrm{C}$ to a depth of $3 \mathrm{~mm}$. After the gel had set, 4-mm wells were cut and removed. The pooled serum of Immunized animals added at the center of plate and extracted LPS of Brucella abortus S99 and also standard LPS of E. coli added at the wells surrounding the central well (containing the serum of immunized animal). Precipitation could appear within $30 \mathrm{~min}$, but final readings were taken at three $h$.

\section{RESULTS}

Rabbit pyrogen test: As the Fig. 1 shows, higher doses of B. abortus S99 LPS causes less or equal body temperature increase in comparison to E. coli LPS doses. According to this graph, $10^{-1} \mathrm{mg} \mathrm{mL}$ of B. abortus S99 LPS caused $6^{\circ} \mathrm{C}$ rise of body temperature following the injection while $10^{-5} \mathrm{mg} \mathrm{mL}^{-1}$ of E. coli LPS increased equal body temperature.

RBT: As the Table 1 shows, sera of immunized animals have been reported positive by RBT while the negative control serum (sample taken before immunization) did not react with rose bengal antigen (agglutination was not observed).

Rapid slide agglutination test: Titer of anti-Brucella antibodies in the sera of immunized animals, taken 2 weeks after the first, second and third immunization reported to be 160,320 and 640 , respectively while no agglutination has been observed in the negative control (Table 2).

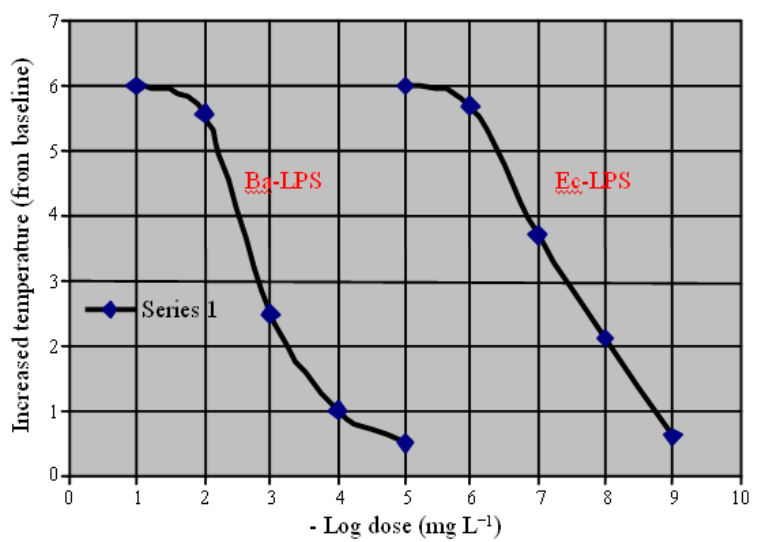

Fig. 1: Rabbit pyrogen test. Increased body temperatures (from the baseline) of animal models injected with $B$. abortus S99 LPS and $E$. coli LPS at the doses indicated. Ba-LPS: B. abortus LPS, Ec-LPS: E. coli LPS 
Am. J. Infect. Dis., 5 (1): 11-16, 2009

Table 1: Results of RBT

\begin{tabular}{|c|c|c|c|c|}
\hline Day of sampling & $\begin{array}{l}0 \\
\text { (Negative control) }\end{array}$ & $\begin{array}{l}14 \text { ( } 2 \text { weeks after the } \\
\text { first immunization) }\end{array}$ & $\begin{array}{l}28 \text { ( } 2 \text { weeks after the } \\
\text { second immunization) }\end{array}$ & $\begin{array}{l}48 \text { ( } 2 \text { weeks after the } \\
\text { third immunization) }\end{array}$ \\
\hline$\overline{\mathrm{RBT}}$ & - & + & + & + \\
\hline
\end{tabular}

Table 2: Results of rapid slide agglutination test

\begin{tabular}{lllll}
\hline Day of sampling & $\begin{array}{l}0 \\
\text { (negative control) }\end{array}$ & $\begin{array}{l}14 \text { (2 weeks after the } \\
\text { first immunization) }\end{array}$ & $\begin{array}{l}28 \text { (2 weeks after the } \\
\text { second immunization) }\end{array}$ & $\begin{array}{l}42(2 \text { weeks after the } \\
\text { third immunization) }\end{array}$ \\
\hline Rapid slide agglutination test & - & 160 & 320 & 640 \\
\hline
\end{tabular}

Table 3: Results of tube agglutination test

\begin{tabular}{lllll}
\hline Day of sampling & 0 (negative control) & $\begin{array}{l}14(2 \text { weeks after the } \\
\text { first immunization })\end{array}$ & $\begin{array}{l}28(2 \text { weeks after the } \\
\text { second immunization })\end{array}$ & $\begin{array}{l}42(2 \text { weeks after the } \\
\text { third immunization })\end{array}$ \\
\hline Tube agglutination test & - & 320 & 640 & 1280 \\
\hline
\end{tabular}

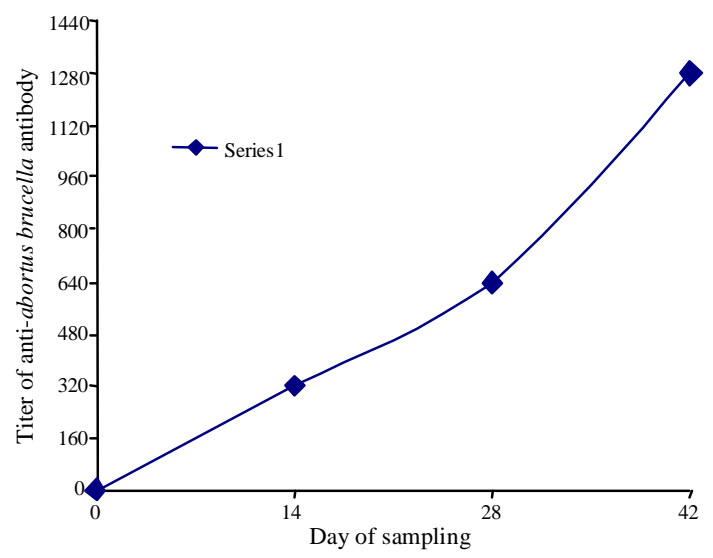

Fig. 2: The Increase of anti- $B$. abortus antibody titer after booster injections (assayed by standard agglutination test)

Standard agglutination test: As the Table 3 shows, no agglutination ring appeared at the bottom of the negative control tube after $24 \mathrm{~h}$. The highest titer of anti-Brucella antibody has been shown in the sample that was taken 2 weeks after the third immunization (1280) while the titer of samples taken 2 weeks after the first and second immunization reported to be 320 and 640, respectively (Fig. 2).

AGID test: The precipitation reaction between the pooled serum of immunized animals and the extracted LPS of B. abortus S99 has been observed after $30 \mathrm{~min}$ but this reaction did not occur between standard LPS of $E$. coli and the pooled serum of immunized animals in the same period of time. Finally after three $h$ a thin line between $E$. coli LPS and the pooled serum appeared, indicating some structural similarities between the LPS of B. abortus S99 and E. coli LPS, while it was thick and sharp between $B$. abortus S99 LPS and the pooled serum because of the specificity of synthesized antibodies against the LPS of B. abortus S99 (Fig. 3).

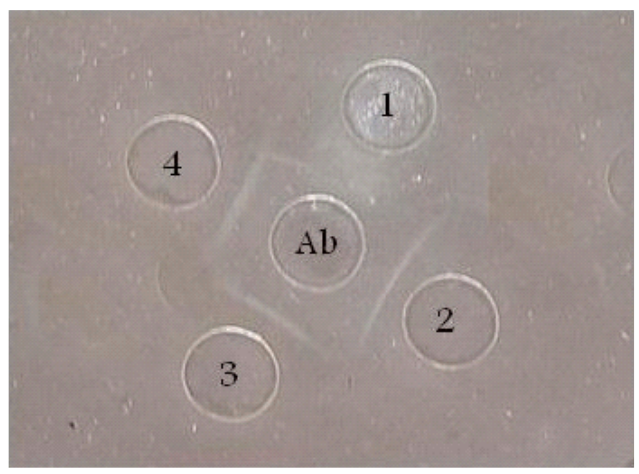

Fig. 3: AGID test confirms the specificity of synthesized antibodies in the animal model for the extracted LPS of B. abortus S99. Also after $3 \mathrm{~h}$ a thin line observed between pooled serum of immunized animals and E. coli LPS. 1: LPS of $E$. coli, 2, 3, 4: LPS of B. abortus S99

\section{DISCUSSION}

Lipopolysaccharide (LPS) is the main expressed antigenic structure at the surface of smooth strains of Brucella abortus. In the present study we analyzed the efficacy of the extracted LPS of Brucella abortus S99 (extracted through our optimized method) ${ }^{[14]}$ to induce the anti-Brucella antibodies in the animal model following the intramuscular injection of this antigen and two booster injections. Sera of all the immunized animals have been detected as positive samples, indicating the potency of this extracted LPS of Brucella abortus S99. The highest antibody titer has been detected 2 weeks after the second injection (demonstrated by tube and slide agglutination methods and has been reported to be 640 and 1280, respectively). Booster injections have efficiently increased the titer of elicited antibodies against Brucella and there was a considerable rise in the assayed titer of analyzed serum samples (Fig. 2). The 
results of Rose Bengal Test (RBT) has been in parallel with Serum agglutination assay results but as RBT is not a quantitative method it may be applied only to detect positive and negative samples and should be followed by agglutination assays to detect the titer of samples.

Currently Serum Agglutination Test (SAT) is the most commonly used and accepted assay to detect the titer of anti-Brucella antibodies ${ }^{[17]}$. Other serology methods such as rose bengal and agarose gel immunodiffusion tests may be applied to distinguish positive and negative serum samples (but not the titer). Since the tube agglutination assay is a time-consuming assay and the process of this method takes at least $24 \mathrm{~h}$, slide (rapid) agglutination assay may be applied to detect the titer of anti-Brucella antibodies in the serum of patients and also in the animal models. In the present study, tube agglutination assay results were in parallel with the slide (rapid) agglutination results but there were one titer difference between these two methods results.

In the present study the specificity of synthesized antibodies in the sera of immunized animals for B. abortus S99 LPS assayed by agarose gelImmunodiffusion method in comparison to $E$. coli LPS. As it is mentioned in the Results, pooled serum of immunize animals react rapidly with $B$. abortus $\mathrm{S} 99$ LPS during the first $30 \mathrm{~min}$ of the test and a sharp precipitation line observed between the wells containing B. abortus S99 LPS and the well containing pooled serum of immunized animals, while no precipitation occurred between the pooled serum and $E$. coli LPS during the same time. Interestingly a thin line of precipitation between pooled serum and E. coli LPS observed after $3 \mathrm{~h}$. Since the animals have been injected with $B$. abortus S99 LPS (not $E$. coli LPS) it could be concluded that this relative reaction may be a sign of some structural similarities between $B$. abortus S99 LPS and E. coli LPS. It is also previously reported that the main structural differences between Brucella and $E$. coli LPS is in the $\mathrm{O}$ chain of these two kind of LPS and other parts of this structure is almost the same in Brucella and E. coli. These structural similarities may lead to a poor cross-reaction between the antibodies elicited against B. abortus S99 LPS with $E$. coli LPS and it seems to be the main reason of reactivity of pooled serum of immunized animals with E. coli LPS in this study.

AGID test has been previously applied to detect positive serum samples of infected dogs and cattle with rough strains of $B$. canis and B. melitensis $16 \mathrm{M}$, respectively and reported to be a sensitive, rapid and reliable method ${ }^{[18]}$. In the present study, we showed that this method would be efficiently used for smooth strains of Brucella such as B. abortus S99.

Rabbit pyrogen test is one of the Invivo assays to evaluate the pyrogenicity of biologic compounds such as LPS and other bacterial antigens. In the present study we evaluated the pyrogenicity of our extracted LPS of B. abortus $\mathrm{S} 99$ in comparison with $E$. coli LPS. As the Fig. 2 shows injection of low doses of E. coli LPS cause higher increase of body temperature in comparison to $B$. abortus LPS which confirms that E. coli LPS is more toxic and pyrogenic than B. abortus S99 LPS. As the graph shows, $10^{-1} \mathrm{mg} \mathrm{mL}^{-1}$ of B. abortus S99 LPS and $10^{-5} \mathrm{mg} \mathrm{mL}^{-1}$ of $E$. coli LPS have equally increased the body temperature following the injection. These data confirm previous study and the fact that Brucella abortus LPS is 10,000 fold less pyrogenic than E. coli LPS ${ }^{[15]}$. Nowadays decreasing the pyrogenicity of immunogenic compounds with microbial origins is one of the strategies to make them applicable for Invivo trials and immunization. Unfortunately, the process of decreasing the pyrogenicity usually leads to less immunogenicity, too. According to this fact, the application of B. abortus S99 LPS for Invivo and immunization aims would be advantageous because of its low pyrogenicity and high potency to induce antibodies against Brucella.

\section{CONCLUSION}

This modified extracted LPS of B. abortus S99 has efficiently promoted the synthesis of high levels of antiBrucella antibodies. Furthermore, elicited antibodies reacted specifically with the extracted LPS which has been intramuscularly administrated to animal models (demonstrated by AGID).

Potency of LPS to induce high titers of specific antibodies against Brucella in parallel with low pyrogenicity of this cellwall structure (defined by rabbi pyrogen test) suggests the possible application of this component as a part of a sub-unit or conjugated vaccine for human brucellosis.

\section{ACKNOWLEDGEMENT}

The researchers would like to thank all colleagues in the Department of Bacterial Vaccines and Antigens Production, Pasteur Institute of Iran, for their kind assistance and advices in the laboratory.

\section{REFERENCES}

1. Corbel, M.J., 1997. Brucellosis: An overview. Emerg. Infect. Dis., 3: 213-221. http://journals.indexcopernicus.com/abstracted.php ?icid $=700746$ 
2. Contreras-Rodriguez, A., B. Ramirez-Zavala, A. Contreras, G. Schurig, N. Sriranganathan and A. Lopez-Merino, 2003. Purification and characterization of an immunogenic aminopeptidase of Brucella melitensis. Infect. Immunol., 71: 5238-5244. DOI: 10.1128/IAI.71.9.5238-5244.2003

3. Orduna, A., A. Almaraz, A. Prado, M.P. Gutierrez, A. Garcia-Pascual, A. Duenas, M. Cuervo, R. Abad, B. Hernandez, B. Lorenzo, M.A. Bratos and A.R. Torres, 2000. Evaluation of an immunocapture-agglutination test (Brucella capt) for serodiagnosis of human brucellosis. J. Clin. Microbiol., 38: 400-4005. www.pubmedcentral.nih.gov/articlerender.fcgi?arti $\mathrm{d}=87532$

4. Yagupsky, P., 1999. Detection of brucellae in blood cultures. J. Clin. Microbiol., 37: 3437-3442. http://jcm.asm.org/cgi/reprint/37/11/3437

5. Yaramis, A., M. Kervancioglu, I. Yildirim, M. Soker, O. Derman and M. Tas, 2004. Severe microangiopathic hemolytic anemia and thrombocytopenia in a child with Brucella infection. Ann. Hematol., 80: 546-548. DOI: 10.1007/s002770100334

6. Miettinen, O. and M. Nurminen, 1985. Comparative analysis of two rates. Stat. Med., 4: 213-226. DOI: 10.1002/sim.4780040211

7. Mantur, G.B. and S.S. Mangalgi, 2004. Evaluation of conventional castaneda and lysis centrifugation blood culture techniques for diagnosis of human brucellosis. J. Clin. Microbiol., 42: 4327-4328. DOI: $10.1128 /$ JCM.42.9.4327-4328.2004

8. Hewitt, W.G. and D.J. Payne, 1984. Estimation of IgG and IgM brucella antibodies in infected and non-infected persons by a radioimmune technique. J. Clin. Pathol., 37: 692-696. DOI: 10.1136/jcp.37.6.692

9. Ariza, J., T. Pellicer, R. Pallare's and F. Gudiol, 1992. Specific antibody profile in human brucellosis. Clin. Infect. Dis., 14: 131-140. http://www.ncbi.nlm.nih.gov/pubmed/1571417

10. Bowden, R.A., G.C. Racaro and P.C. Baldi, 1999. Effect of early antibiotic treatment on the antibody response to cytoplasmic proteins of Brucella melitensis in Mice. Clin. Vac. Immunol., 6: 440-443.

http://cvi.asm.org/cgi/content/full/6/3/440

11. Singh, M., M. Salaria and L. Kumar, 2007. Penumonic presentation of brucellosis. Ind. J. Pediat., 72: 65-66. DOI: 10.1007/BF02760583
12. Cherwonogrodzky, J.W. and K.H. Nielsen, 1998. Brucella abortus 1119-3 0-Chain polysaccharide to differentiate sera from $B$. abortus S-19-vaccinated and field-strain-infected cattle by agar gel immunodiffusion. J. Clin. Microbiol., 26: 1120-1123. http://www.ncbi.nlm.nih.gov/pubmed/3133389

13. Salmani, A.S., S.D. Siadat, H. Ahmadi, M. Nejati, D. Norouzian, B. Tabaraie, M. Abbasi, M. Karbasian, A.M. Mobarez and R. Shapouri, 2008. Optimization of Brucella abortus S99 lipopolysaccharide extraction by phenol and butanol methods. Res. J. Biol. Sci., 3: 576-580. http://www.medwelljournals.com/fulltext/rjbs/2008 1576-580.pdf

14. Shapouri, R., A.M. Mobarez, H. Ahmadi, B. Tabaraie, R. Hosseini Doust, D. Norouzian, A. Zavaran Hosseini and S.D. Siadat, 2008. Optimization of Brucella abortus fermenter culture conditions and LPS extraction method for antigen production. Res. J. Microbiol., 3: 1-8. http://www.academicjournals.net/fulltext/jm/2008/ 1-8.pdf

15. Goldstein, J., Th. Hoffman, C. Frasch, E.F. Lizzio, B.R. Beining, D. Hochstein, Y.L. Lim, R.D. Angus and B. Golding, 1992. Lipopolysaccharide from Brucella abortus is less toxic than that from Escherichia coli, suggesting the possible use of $B$. abortus or LPS from B. abortus as a carrier in vaccines. Infect. Immunol., 60: 1385-1389. http://iai.asm.org/cgi/reprint/60/4/1385.pdf

16. Alton, G.G, L.M. Jones, R.D. Angus and J.M. Verger, 1988. Techniques for the brucellosis laboratory. Vet. Res. Commun., 13: 420-420. DOI: 10.1007/BF00402562.

17. Baum, M., O. Zamir, R. Bergman-Rios, E. Katz, Z. Beider, A. Cohen and M. Banai, 1995. Comparative evaluation of microagglutination test and serum agglutination test as supplementary diagnostic methods for brucellosis. J. Clin. Microbiol., 33: 2166-70. http://www.pubmedcentral.nih.gov/picrender.fcgi? artid=228357\&blobtype $=$ pdf

18. Myers, D.M. and V.M. Varela-Díaz, 1974. Comparative sensitivity of gel-diffusion and the tube agglutination tests for the detection of Brucella canis antibodies in experimentally infected dogs. Applied Microbiol., 28: 1-4. http://aem.asm.org/cgi/content/abstract/28/1/1 